\title{
Article
}

\section{The T Cell Repertoires from Nickel Sensitized Joint Implant Failure Patients}

\author{
Lan Chen ${ }^{1}$, Yan Zhang ${ }^{1}{ }^{(D)}$, Karin Pacheco ${ }^{2}$ and Shaodong Dai ${ }^{1, *}$ \\ 1 Skaggs School of Pharmacy and Pharmaceutical Sciences, University of Colorado Anschutz Medical Campus, \\ Aurora, CO 80045, USA; lan.chen@cuanschutz.edu (L.C.); yan.zhang@cuanschutz.edu (Y.Z.) \\ 2 Department of Medicine, National Jewish Health, Denver, CO 80206, USA; PachecoK@NJHealth.org \\ * Correspondence: shaodong.dai@cuanschutz.edu
}

check for updates

Citation: Chen, L.; Zhang, Y.; Pacheco, K.; Dai, S. The T Cell Repertoires from Nickel Sensitized Joint Implant Failure Patients. Int. J. Mol. Sci. 2021, 22, 2428. https:// doi.org/10.3390/ijms22052428

Received: 31 January 2021

Accepted: 23 February 2021

Published: 28 February 2021

Publisher's Note: MDPI stays neutral with regard to jurisdictional claims in published maps and institutional affiliations.

Copyright: (C) 2021 by the authors Licensee MDPI, Basel, Switzerland. This article is an open access article distributed under the terms and conditions of the Creative Commons Attribution (CC BY) license (https:/ / creativecommons.org/licenses/by/ $4.0 /)$.

\begin{abstract}
Nickel $\left(\mathrm{Ni}^{2+}\right)$ is one of the most common allergens, affecting around $10-15 \%$ of the general population. As the demand for orthopedic implant surgery rises, the number of surgical revisions due to joint implant failure also increases. There is evidence that some patients develop joint failure due to an immune response to a component of the implant, and we have found that $\mathrm{Ni}^{2+}$ is an especially important cause. Hence, understanding the mechanisms by which $\mathrm{Ni}^{2+}$ allergy induces joint implant failure becomes a critical research question. The structural basis of $\mathrm{Ni}^{2+}$ activation of pathogenic $\mathrm{T}$ cells is still not clear. The purpose of this study was to characterize $\mathrm{Ni}^{2+}$-reactive $\mathrm{T}$ cell repertoires derived from the peripheral blood of joint failure patients due to $\mathrm{Ni}^{2+}$ sensitization using single-cell sequencing techniques. We stimulated the proliferation of $\mathrm{Ni}^{2+}$-reactive $\mathrm{T}$ cells from two implant failure patients in vitro, and sorted them for single-cell VDJ sequencing $(10 \times$ genomics). We identified 2650 productive V-J spanning pairs. Both TCR $\alpha$ chains and $\beta$ chains were enriched. TRBV18 usage is the highest in the P7 CD4+ population (18.1\%), and TRBV5-1 usage is the highest in the P7 CD8+ population (12.1\%). TRBV19 and TRBV20-1 segments are present in a high percentage of both P7 and P9 sequenced T cells. Remarkably, the alpha and beta chain combination of TRAV41-TRBV18 accounts for $13.5 \%$ of the CD4+ population of P7 patient. Compared to current Ni specific $\mathrm{T}$ cell repertoire studies of contact dermatitis, the $\mathrm{V} \alpha$ and $\mathrm{V} \beta$ usages of these joint implant failure patients were different. This could be due to the different availability of self-peptides in these two different tissues. However, TRBV19 (V $\beta 17)$ was among frequently used TCR $\beta$ chains, which are common in previous reports. This implies that some pathogenic T cells could be similar in $\mathrm{Ni}^{2+}$ hypersensitivities in skin and joints. The alignment of the TCR CDR3 $\beta$ sequences showed a conserved glutamic acid (Glu) that could potentially interact with $\mathrm{Ni}^{2+}$. The study of these $\mathrm{Ni}^{2+}$ specific TCRs may shed light on the molecular mechanism of T cell activation by low molecular weight chemical haptens.
\end{abstract}

Keywords: metal hapten; hypersensitivity; single-cell sequencing; joint implant failure; TCR repertoire

\section{Introduction}

There were approximately 5.2 million total knee arthroplasties performed in the United States during the 2000-2010 period [1], and the demand for total hip and knee arthroplasties is predicted to grow to 850,000 and 1.91 million, respectively, by 2030 [2]. Total joint replacement constitutes the highest cost for Medicare programs, and similar demand is growing worldwide [3,4]. The majority of joint replacements are performed for osteoarthritis, increasingly common in an aging world population, with a history of sports induced injuries and rising rates of obesity. Other, less common causes include connective tissue disease destruction of large joints, avascular necrosis caused by injury to vascular joint structures, and treatment with steroids, with smaller contributions from fractures, benign and malignant bone tumors, and hemarthrosis (https:/ / orthoinfo.aaos.org/en/ treatment/total-joint-replacement/). 
Joint replacement prosthetic manufacturing is dominated by a few large companies, although there are smaller companies providing specialized implants. The majority of commercially available implants are composed of a cobalt/chromium alloy containing $60 \%$ cobalt, $30 \%$ chromium, $5 \%$ molybdenum, with from 0.5 to $2 \%$ nickel. Stainless steel, commonly used in orthopedic screws and staples, contains from 13 to $15.5 \%$ nickel, from 12 to $18 \%$ chromium, from 0.8 to $1.8 \%$ manganese, and the balance of iron. All these metals, with the exception of iron, can cause sensitization and disease in humans, most frequently nickel, cobalt, and chromium. The most dominant of these is nickel.

Generally, up to $90 \%$ of joint replacements do well, and provide significant health and socioeconomic benefits to the recipients. About $10 \%$ of these, however, will fail, due to infection, mechanical issues, and immune mediated responses to the implant [5]. All metal implants undergo corrosion in synovial fluid, and release metal ions that can act as haptens to activate the immune system [6]. As the number of arthroplasties increases worldwide, the incidence of implant failure caused by $\mathrm{Ni}^{2+}$ hypersensitivity reactions will grow concomitantly.

Long term sensitization to $\mathrm{Ni}^{2+}$ is known to be a type IV cell-mediated hypersensitivity. This process can be induced by $\mathrm{Ni}^{2+}$ binding to the TLR4 receptor [7] on antigen presenting cells (APCs) such as dendritic cells, activating them, and eliciting the secretion of proinflammatory cytokines. APCs then carry the $\mathrm{Ni}^{2+}$ antigen to regional lymph nodes, where they prime naive $\mathrm{T}$ lymphocytes to differentiate into effector $\mathrm{T}$ cells, and begin the process of antigen-specific $\mathrm{T}$ cell activation [8]. $\alpha \beta \mathrm{T}$ cells commonly recognize peptide antigens bound to MHCI and MHCII molecules, and play a central role in adaptive immunity. MHC molecules can also present small molecules in addition to peptides to T cells. However, the specific molecular basis of $\mathrm{T}$ cell activation by $\mathrm{Ni}^{2+}$ ions still remains unclear.

Although studies of joint implant failure have established the association with $\mathrm{Ni}^{2+}$ hypersensitivity, the dominant TCRs responsible for the process are still unknown. Most previous studies of the molecular mechanisms of $\mathrm{Ni}^{2+}$ hypersensitivity have mainly focused on $\mathrm{Ni}^{2+}$-reactive $\mathrm{T}$ cells from contact dermatitis patients. Here, the antigen presenting cells are Langerhans cells and dermal dendritic cells, which differ from the macrophages and dendritic cells relevant in the joint, such that the $\mathrm{Ni}^{2+}$ response in the joint could be totally different from the dermal response $[9,10]$.

In contact dermatitis subjects, isolation of a panel of $\mathrm{Ni}^{2+}$-specific $\mathrm{T}$ cell clones from peripheral blood mononuclear cells (PBMCs) by the Weltzien group identified that TRBV19 (VB17) TCRs predominated [11,12]. One of the TRBV19 CD4+ TCR, SE9, was MHC and peptide promiscuous [13]. The other TRBV19 TCR, ANi2.3, was strictly DR52c restricted, requiring an unknown endogenous peptide to effectively present $\mathrm{Ni}^{2+}$ ions [14]. We identified a panel of $\mathrm{Ni}^{2+}$ independent mimotopes that can directly activate the ANi2.3 TCR independent of $\mathrm{Ni}^{2+}$ [15]. The ternary structure of the ANi2.3 TCR, MHC and mimotope confirmed that $\mathrm{Ni}^{2+}$ presentation required both a specific MHC and peptides [15]. Recently, a study by Bechara et al. also showed the dominant usage of TRBV19 in highly expanded clones from $\mathrm{Ni}^{2+}$-specific naive $\mathrm{T}$ cell lines derived from $\mathrm{Ni}^{2+}$ sensitized contact dermatitis subjects [16]. However, another paper found dominant usage of TRAV9-2 rather than TRBV19 in CD154 positive nickel-stimulated T cells [17]. The ternary complex structure of the ANi2.3 TCR, DR52C and $\mathrm{Ni}^{2+}$ dependent mimotope was determined. However, it is still not clear as to how the unknown endogenous peptide presents $\mathrm{Ni}^{2+}$ to the ANi2.3 TCR, or whether the TRBV19 TCR is the dominant TCR that also induces joint implant failure due to $\mathrm{Ni}^{2+}$ sensitization. We previously found that around $1 \%$ of peripheral blood $\mathrm{T}$ cells from several Ni-sensitized joint failure patients interact with the DR52c-mimotope. In addition, the percentage of TRBV19 CD4 T cells increases from $0.76 \%$ to $13.3 \%$ when stimulated with $\mathrm{Ni}^{2+}$ in vitro [18]. This suggests that the TRBV19 TCR may also play an important role in inducing $\mathrm{Ni}^{2+}$ sensitization in periprosthetic tissue after arthroplasty.

In this study, a $10 \times$ single cell VDJ sequencing technique was utilized to analyze the $\mathrm{Ni}^{2+}$-reactive $\mathrm{T}$ cell repertoire derived from the PBMCs from two joint failure patients. We identified several highly expanded $\mathrm{T}$ cell clones that preferentially used specific TCR 
alpha and beta chains. Furthermore, the alignment of the CDR3 $\beta$ sequences of these TCRs shows a conserved Glu at the last fourth position that could directly interact with $\mathrm{Ni}^{2+}$ ions. Given the rising number of joint replacements using nickel containing implants worldwide, and the concomitant rise in failure due to nickel sensitization, these results provide an important insight in this process, as well as suggestions for therapeutic interventions.

\section{Results}

\subsection{Characterization of Subject Clinical Features}

Out of a population of $n=483$ implant failure patients, $29 \%$ were sensitized to $\mathrm{Ni}^{2+}$, peripheral blood was selected from two female joint implant failure patients, designated P7 and P9. Both patients were post implant: P7 with a left total hip arthroplasty with a mixed, CoCrMo and Titanium alloy implant; P9 had a right total knee arthroplasty with a CoCrMo alloy implant and bone cement. Both patients were $\mathrm{Ni}^{2+}$ sensitized based on patch testing, along with a strongly positive $\mathrm{Ni}^{2+}$ lymphocyte proliferation test (NiLPT) stimulation index. More importantly, when stimulated with $\mathrm{Ni}^{2+}$, both PBMCs demonstrated a clear population that stained with a tetramer of the DR52c/ $\mathrm{Ni}^{2+}$ mimotope [10]. The clinical data summary is shown in Table 1. PBMCs were separated from fresh blood and stored in liquid nitrogen. DR genes were identified by high resolution methods: P7 is positive for DR4, DR7, and DR53, whereas P9 is positive for DR7, DR11, DR53, and DR52b.

Table 1. Summary of clinical data from the two subjects.

\begin{tabular}{cccccc}
\hline Patient & HLA Typing & Joint Replaced & $\begin{array}{c}\text { Size of Nickel Patch } \\
\text { Test } \\
\text { (Range is 1+ to 3+ ) }\end{array}$ & Nickel LPT PSI \\
\hline 7 & $\begin{array}{r}\text { DRB1*04:01:01, } \\
\text { DRB1*07:01P }\end{array}$ & DRB4*01:BRF & Postop L THA failure & $1+$ & 12.2 \\
\hline 9 & $\begin{array}{r}\text { DRB1*07:01P, } \\
\text { DRB1*11:01:02 }\end{array}$ & DRB3*02:ANCDJ & DRB4*01:BRF & Postop R TKA failure & $3+$ \\
\hline
\end{tabular}

\subsection{PBMCs Proliferated and Secreted IFN $\gamma$ with $\mathrm{Ni}^{2+}$ Stimulation In Vitro}

PBMCs from P7 and P9 patients were stimulated with $\mathrm{Ni}^{2+}$ and cultured for three weeks in vitro (Figure 1a). During the first week, PBMCs were cultured in media with $100 \mu \mathrm{M} \mathrm{NiSO}_{4}$ to support $\mathrm{Ni}^{2+}$-specific $\mathrm{T}$ cell proliferation. During the second week, the media was changed to Ni-free, and hIL2 was added to maintain cell viability. During the third week, media was changed to hIL2 free, with the addition of $100 \mu \mathrm{M} \mathrm{NiSO}_{4}$ and irradiated PBMCs as APCs were added to encourage proliferation of $\mathrm{Ni}^{2+}$-specific T cells. Cells were labeled with CellTrace proliferation dye at the beginning of the third week, to enable separation of the proliferating cells based on the fluorescence at the end of the experiment. To monitor $\mathrm{T}$ cell stimulation, culture supernatant was collected every three or four days for IFN $\gamma$ measurement. IFN $\gamma$ was selected as a proinflammatory cytokine marker that is pivotal in antiviral activity and autoinflammatory diseases, and is usually secreted by differentiated Th1 CD4+ and Tc1 CD8+ cells. Both samples demonstrated a trend of increased IFN $\gamma$ secretion (Figure 1b), although P7 showed a fall in IFN $\gamma$ from the 17 th to the 21st day.

At the end of the 3-week stimulation, all cells were harvested, and proliferating cells were separated based on incorporation of the CellTrace proliferation dye (Figure 1c,d). For both P7 and P9, three separate cell populations were identified based on cell size and granularity. Live and proliferating lymphocytes are located in population 3 circled in $1 \mathrm{c}$ and $1 \mathrm{~d}$. Based on their size and granularity, populations 1 and 2 contained predominantly dead or dying cells not labeled with CellTrace. The proliferating cells from populations 2 and 3 were collected, labeled with totalseqC hashtag antibodies, and sent for single cell $\mathrm{V}(\mathrm{D}) \mathrm{J}$ sequencing and single cell gene expression sequencing. 
(a)
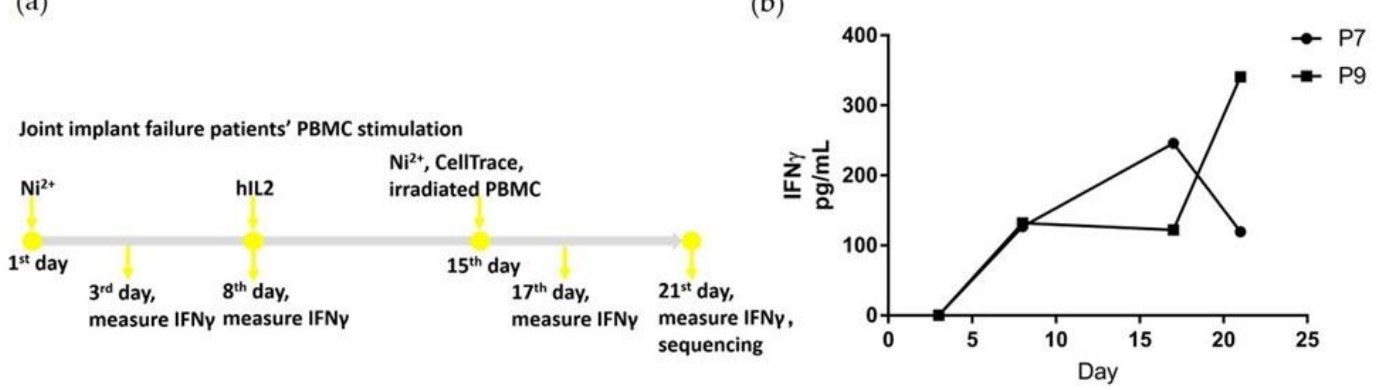

(d) (c)

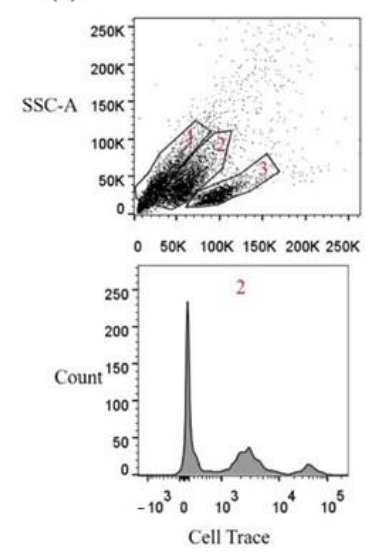

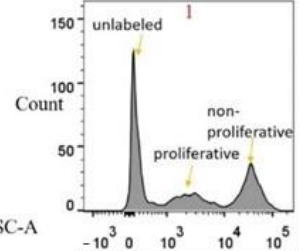

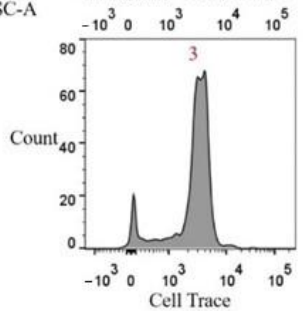

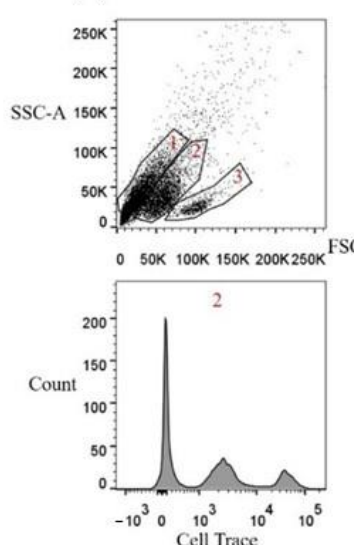

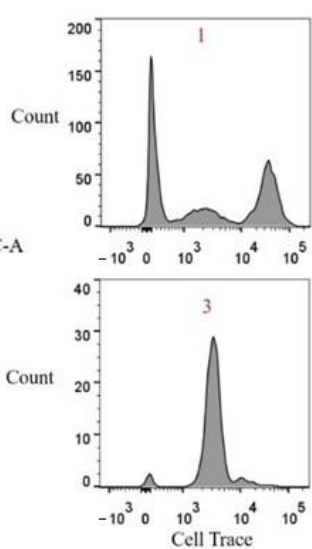

Figure 1. $\mathrm{Ni}^{2+}$ treated PBMCs include proliferating cells after three weeks in vitro culture. (a) shows the stimulation protocol for PBMCs derived from joint implant failure patients; (b) levels of secreted IFN $\gamma$ in the culture media; (c) and (d) flow cytometry results of the harvested PBMCs at the last day of stimulation for P7 and P9, respectively. Based on the size and scattering, cells were separated into three populations. We examined the CellTrace dye fluorescence for each of the three populations separately. The "unlabeled", "proliferative", and "non-proliferative" peaks are indicated in (c) 1 population, and similar in the other panels. The proliferative cells in populations 2 and 3 were collected for single cell sequencing.

\subsection{Preferential TCR Usage in the Repertoire of $\mathrm{Ni}^{2+}$-Recognizing $\mathrm{CD} 4+$ and $\mathrm{CD} 8+\mathrm{T}$ Cells}

Single-cell VDJ sequencing yielded 3682 individual clones out of $\sim 5000$ that were sequenced, and 2650 of them included productive V-J spanning pairs. After demultiplexing hashtag results, we were able to analyze the paired TCR sequences for P7 CD4+, P7 CD8+, P9 CD4+, and P9 CD8+ cells. The TRBV and TRBJ usage percentages for these T cell types are shown in Figure 2, and the TRAV and TRAJ usage percentages are shown in Figure 3. Both TCR alpha and beta chains showed preferential usage. Noticeably, TRBV18 usage is the highest in the P7 CD4+ population (18.1\%) but not in the other three populations; TRBV5-1 usage is the highest in the P7 CD8+ population (12.1\%), but also found in a high percentage of the other three cell populations (Figure 2a). TRBV19 and TRBV20-1 are present in a high percentage of both P7 and P9 sequenced T cells; TRBV19 accounts for $5.3 \%$ of P7 and $8 \%$ of P9 CD4+ T cells, and TRBV20-1 accounts for $6.4 \%$ of P7 and $7.5 \%$ of P9 CD4+ T cells. As to TRBJ usage, TRBJ1-2, TRBJ2-1, and TRBJ2-7 TCR motifs are the most highly used in all four populations (Figure $2 b$ ).

As to TRAV usage (Figure 3a,b), TRAV13-1 is the most highly expanded in both CD4+ CD8+ in both patients $(6.8 \%, 10.5 \%, 7.6 \%$, and $8.1 \%$, respectively); TRAV41 constituted $11.2 \%$ of the P7 CD4+ population but was not found in a high frequency in the other populations; TRAV29DV5 comprised $10.5 \%$ of the P7 CD8+ population; TRAJ57 was found preferentially in the P7 CD4+ population (12.4\%) (Figure 3b); other TRAJ sequences were not preferentially used. 
(a)

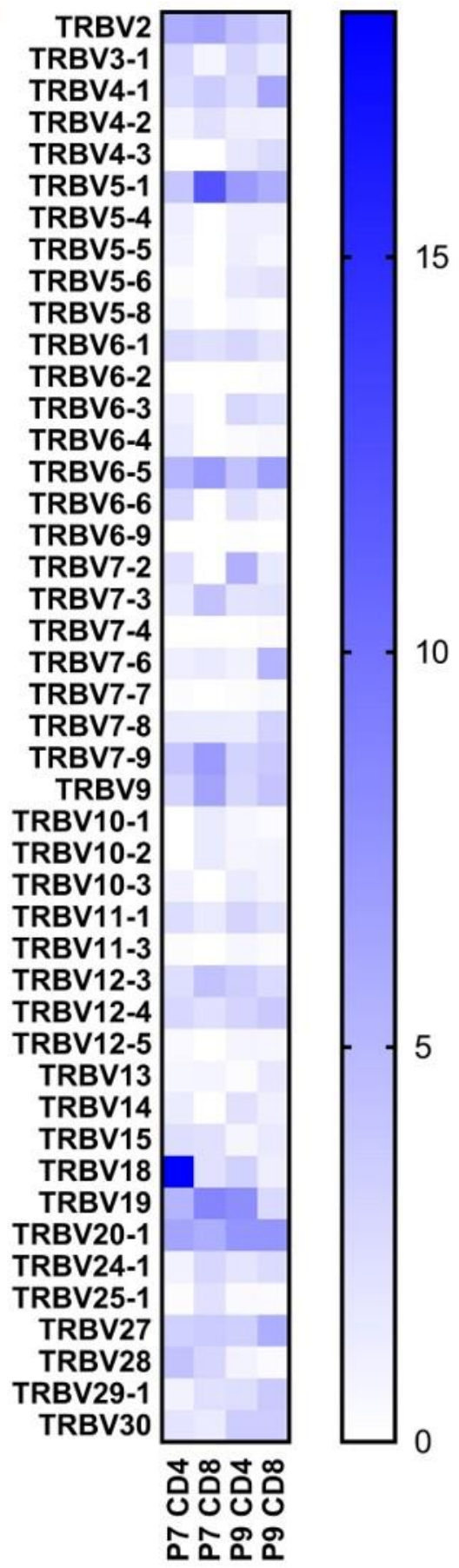

(b)

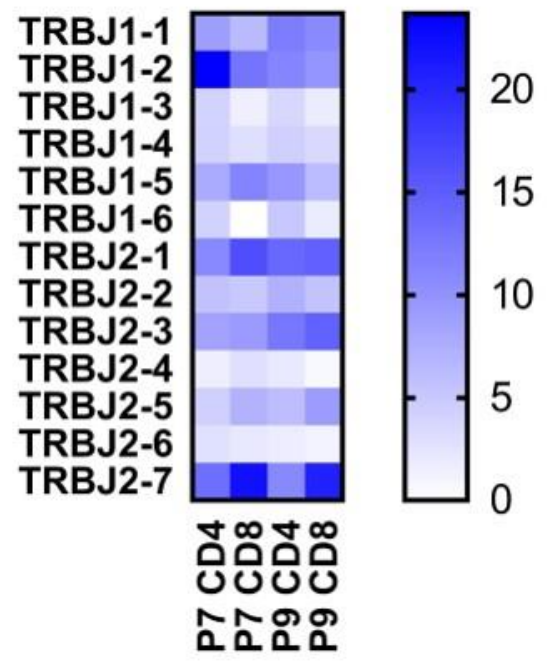

Figure 2. TRBV and TRBJ usage in $\mathrm{Ni}^{2+}$-reactive $\mathrm{CD} 4+$ and $\mathrm{CD} 8+\mathrm{T}$ cell clones in $\mathrm{P} 7$ and $\mathrm{P} 9$ subjects. (a) Percentage of the TRBV gene usage. (b) Percentage of the TRBJ gene usage. All gene names are in IMGT nomenclature. The scale reflects the percentage present in each designated population.

\subsection{Highly Expanded TCR Clones Are Detected in Both CD4+ and CD8+T Cells}

The most highly expanded clones in two samples for both CD4+ and CD8+ cells are listed in Table 2. The alpha and beta chain combination of TRAV41-TRBV18 accounts for $13.5 \%$ of the P7 CD4+ population, but is not found in the other populations. Remarkably, the TRBV19 TCR accounts for $9.0 \%$ of the P9 CD4+ cells, and 1/3 of the TRBV19 T cell population is joined to the TRBJ1-5 sequence. Although $\mathrm{Ni}^{2+}$-reactive $\mathrm{CD} 8+\mathrm{T}$ cells have 
not been identified in previous studies, we found some highly expanded CD8+ T cell clones for both subjects, for example, TRAV29DV5-TRBV6-5 in P7 and TRAV22-TRBV7-6 in P9.

The hashtag labeling of sorted cells enabled us to calculate the relative percentages of $\mathrm{CD} 4+$ and CD8+ T cells for both subjects, and both have similar ratios. CD4+ and CD8+ T cells constituted $79.2 \%$ and $20.8 \%$, respectively, for P7; and $76.2 \%$ and $23.8 \%$, respectively, for P9 (Figure 4).

(a)

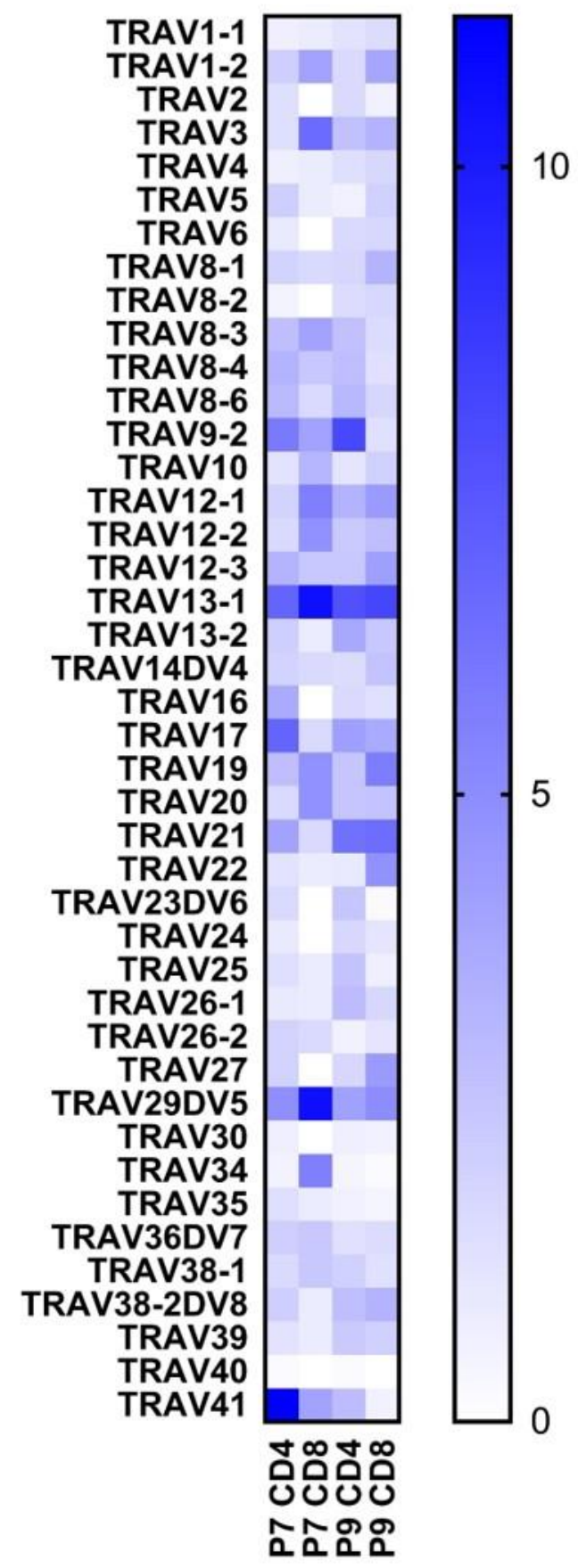

(b)

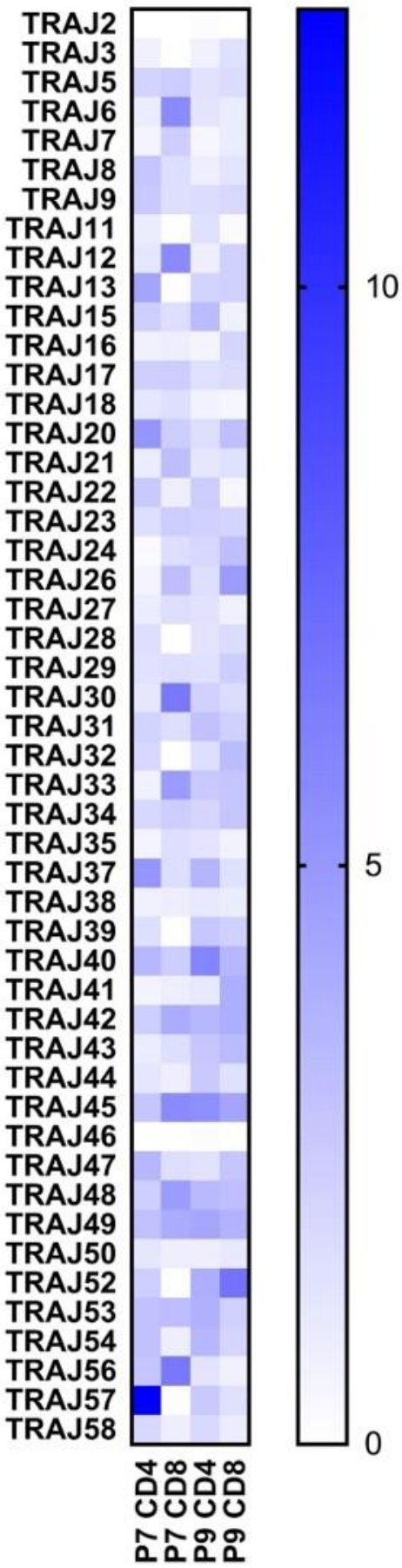

Figure 3. TRAV and TRAJ usage in $\mathrm{Ni}^{2+}$-reactive $\mathrm{CD} 4+$ and $\mathrm{CD} 8+\mathrm{T}$ cell clones in subjects $\mathrm{P7}$ and P9. (a) Percentage of the TRAV gene usage. (b) Percentage of the TRAJ gene usage. All the gene names are in IMGT nomenclature. The scale shows the percentage use in each of the four designated populations. 
Table 2. Most frequent pairs of V-J usage sequenced.

\begin{tabular}{|c|c|c|c|c|c|}
\hline Number of Clones & Percentage & TRAV & TRAJ & TRBV & TRBJ \\
\hline \multicolumn{6}{|l|}{ P7 CD4+ } \\
\hline 51 & $13.49 \%$ & TRAV41 & TRAJ57 & TRBV18 & TRBJ1-2 \\
\hline 8 & $2.12 \%$ & TRAV16 & TRAJ13 & TRBV15 & TRBJ2-7 \\
\hline 4 & $1.06 \%$ & TRAV13-1 & TRAJ45 & TRBV12-3 & TRBJ1-1 \\
\hline 3 & $0.79 \%$ & TRAV5 & TRAJ22 & TRBV5-1 & TRBJ2-1 \\
\hline 3 & $0.79 \%$ & TRAV17 & TRAJ47 & TRBV2;TRBV6-4 & TRBJ2-6;TRBJ1-1 \\
\hline 3 & $0.79 \%$ & TRAV13-1 & TRAJ9 & TRBV28 & TRBJ1-2 \\
\hline 2 & $0.53 \%$ & TRAV1-2 & TRAJ18 & TRBV9 & TRBJ2-1 \\
\hline 2 & $0.53 \%$ & TRAV19 & TRAJ17 & TRBV7-3 & TRBJ2-2 \\
\hline 2 & $0.53 \%$ & TRAV29DV5 & TRAJ48 & TRBV7-9 & TRBJ1-2 \\
\hline 2 & $0.53 \%$ & TRAV8-1 & TRAJ37 & TRBV6-5 & TRBJ2-7 \\
\hline \multicolumn{6}{|l|}{ P7 CD8+ } \\
\hline 9 & $7.96 \%$ & TRAV29DV5 & TRAJ56 & TRBV6-5 & TRBJ1-5 \\
\hline 5 & $4.42 \%$ & TRAV34 & TRAJ26 & TRBV5-1 & TRBJ2-7 \\
\hline 4 & $3.54 \%$ & TRAV3 & TRAJ30 & TRBV2 & TRBJ2-3 \\
\hline 4 & $3.54 \%$ & TRAV41 & TRAJ49 & TRBV9 & TRBJ2-7 \\
\hline 3 & $2.65 \%$ & TRAV10 & TRAJ12 & TRBV19 & TRBJ2-1 \\
\hline 3 & $2.65 \%$ & TRAV26-2 & TRAJ48 & TRBV20-1 & TRBJ2-7 \\
\hline 3 & $2.65 \%$ & TRAV12-2 & TRAJ8 & TRBV24-1 & TRBJ2-5 \\
\hline 2 & $1.77 \%$ & TRAV13-1 & TRAJ21 & TRBV7-3 & TRBJ2-5 \\
\hline 2 & $1.77 \%$ & TRAV8-4 & TRAJ49 & TRBV7-9 & TRBJ1-1 \\
\hline 2 & $1.77 \%$ & TRAV13-1 & TRAJ6 & TRBV10-1 & TRBJ2-7 \\
\hline 2 & $1.77 \%$ & TRAV12-3 & TRAJ18 & TRBV7-9 & TRBJ2-4 \\
\hline \multicolumn{6}{|l|}{ P9 CD4+ } \\
\hline 24 & $1.47 \%$ & $\begin{array}{c}\text { TRAV13- } \\
\text { 1;TRAV23DV6 }\end{array}$ & TRAJ40;TRAJ15 & TRBV19 & TRBJ1-5 \\
\hline 18 & $1.10 \%$ & TRAV23DV6 & TRAJ15 & TRBV19 & TRBJ1-5 \\
\hline 12 & $0.73 \%$ & TRAV13-2 & TRAJ45 & TRBV6-3 & TRBJ1-1 \\
\hline 11 & $0.67 \%$ & TRAV13-2;TRAV25 & TRAJ45;TRAJ37 & TRBV6-3 & TRBJ1-1 \\
\hline 9 & $0.55 \%$ & TRAV39 & TRAJ44 & TRBV7-2 & TRBJ2-3 \\
\hline 7 & $0.43 \%$ & TRAV13-1 & TRAJ40 & TRBV19 & TRBJ1-5 \\
\hline 6 & $0.37 \%$ & TRAV8-2 & TRAJ40 & TRBV27 & TRBJ2-5 \\
\hline 6 & $0.37 \%$ & TRAV9-2 & TRAJ31 & TRBV27 & TRBJ2-7 \\
\hline 5 & $0.31 \%$ & TRAV3 & TRAJ23 & TRBV19 & TRBJ1-2 \\
\hline 5 & $0.31 \%$ & TRAV9-2 & TRAJ57 & TRBV14 & TRBJ1-1 \\
\hline \multicolumn{6}{|l|}{ P9 CD8+ } \\
\hline 19 & $3.97 \%$ & TRAV22 & TRAJ52 & TRBV7-6 & TRBJ2-7 \\
\hline 9 & $1.88 \%$ & TRAV8-1 & TRAJ5 & TRBV5-1 & TRBJ2-3 \\
\hline 8 & $1.67 \%$ & TRAV12-3 & TRAJ26 & TRBV7-3 & TRBJ2-3 \\
\hline 6 & $1.26 \%$ & TRAV4 & TRAJ20 & TRBV24-1 & TRBJ2-5 \\
\hline 5 & $1.05 \%$ & TRAV27 & TRAJ29 & TRBV20-1 & TRBJ2-1 \\
\hline 5 & $1.05 \%$ & TRAV36DV7 & TRAJ24 & TRBV20-1 & TRBJ1-2 \\
\hline 5 & $1.05 \%$ & TRAV29DV5 & TRAJ33 & TRBV7-8 & TRBJ1-1 \\
\hline 5 & $1.05 \%$ & TRAV13-1 & TRAJ32 & TRBV12-3 & TRBJ2-5 \\
\hline 5 & $1.05 \%$ & TRAV21 & TRAJ45 & TRBV6-5 & TRBJ1-5 \\
\hline 4 & $0.84 \%$ & TRAV12-1;TRAV20 & TRAJ41;TRAJ49 & TRBV6-5 & TRBJ2-7 \\
\hline
\end{tabular}




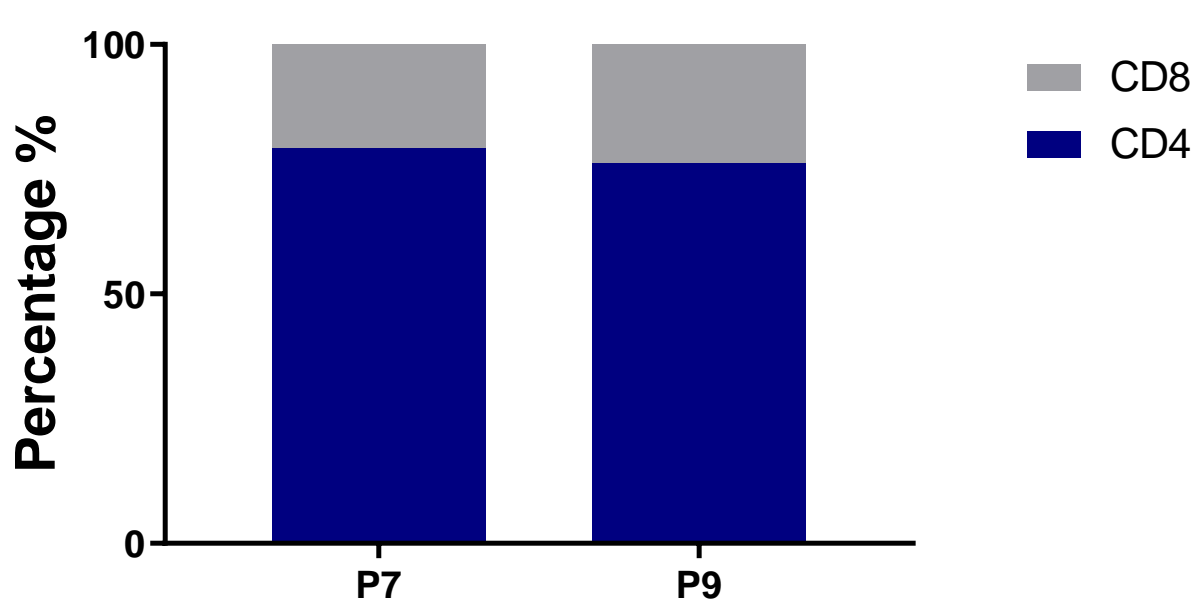

Figure 4. Percentage of CD4+ and CD8+ T cells in sequenced samples.

\subsection{Conserved Glu Is Found in the CDR3 Sequences}

Previous studies have indicated that TCR beta chains have the most contact with the antigen peptide [15], and the changes in $\mathrm{V} \beta$ repertoire usage correspond to the $\mathrm{Ni}^{2+}$ response [19-21]. As the CDR3 region is the most variable and important for direct antigen interaction, we aligned the sequences of CDR3 $\beta$ from both P7 and P9 TCRs. It is noticeable that Glu is conserved at the last 4th position of CDR3 (Figure 5a) in both subjects, which could be the important amino acid to interact with the positively charged $\mathrm{Ni}^{2+}$. The CDR3 $\beta$ lengths of both P7 and P9 shared similar size distributions, and the TCR from CD4+ and CD8+ cells both peaked at 13-15 amino acid length (Figure 5b). V $\beta$ Segments seemed to skew towards long CDR3s comparing to health controls [22]. This would suggest that CDR3 $\mathrm{Ni}^{2+}$ coordination would require a long flexible loop as most metal binding sites in proteins. Indeed, the CDR3 $\beta$ of $\mathrm{Ni}^{2+}$ specific TCR Ani2.3 contained 16 amino acids, and adopted a highly flexible and extended confirmation in the crystal structure [15].

(a)
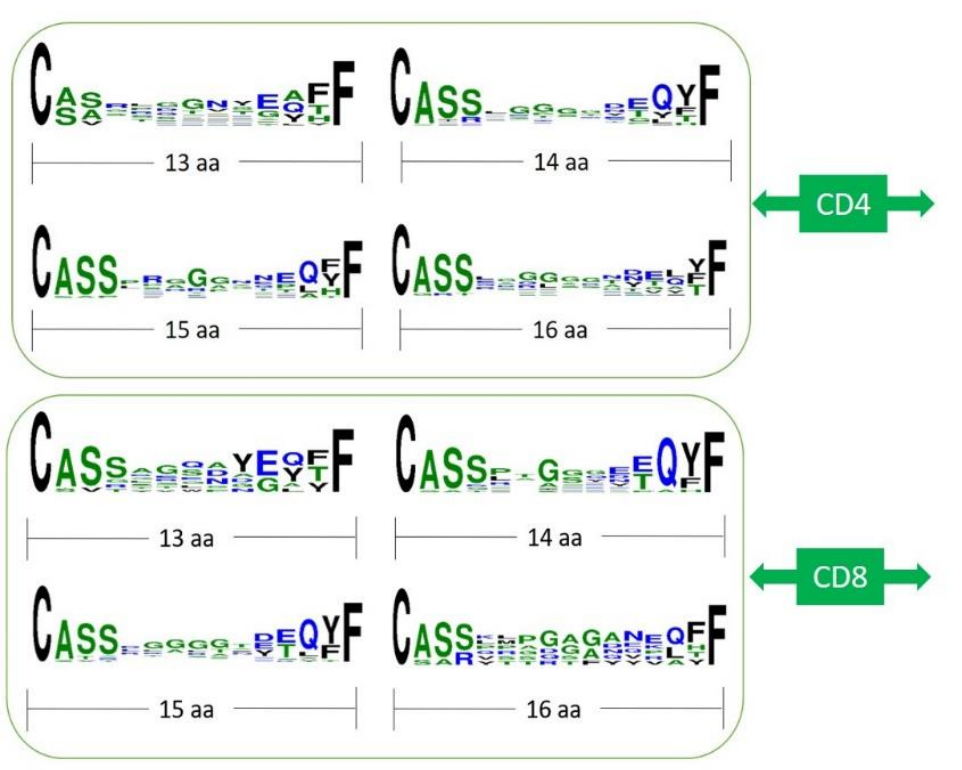

(b)
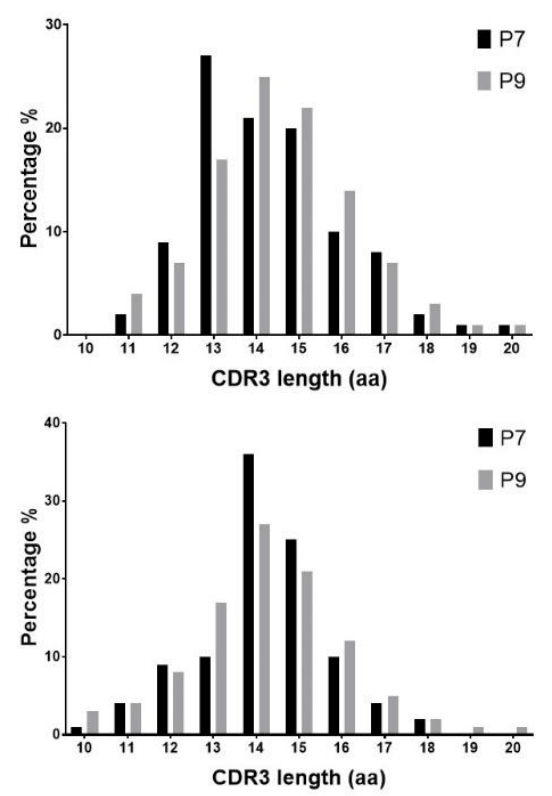

Figure 5. CDR3 $\beta$ sequence alignment and length comparison: (a) shows the alignment of CDR3 $\beta$ sequences from P7 and P9 T cells combined using Weblogo; (b) shows the distribution of the CDR3 $\beta$ length of P7 and P9 T cells, respectively, in CD4+ (top) and CD8+ (bottom) T cells. The $x$-axis is the length from amino acid C to $\mathrm{F}$, and the $y$-axis is the percentage. 


\section{Discussion}

A growing number of studies demonstrate that nickel allergy not only leads to mild to severe contact dermatitis, but also contributes to joint replacement failure. Several clinical studies have documented the contribution of metal allergy, and especially nickel allergy, to joint replacement failure [5,10,23-26]. Some other clinical studies have also demonstrated improvement in nickel allergy patients once their $\mathrm{Ni}^{2+}$ containing implant is replaced with non- $\mathrm{Ni}^{2+}$ hardware, compared to those $\mathrm{Ni}^{2+}$ sensitized implant failure patients who are not revised and remain symptomatic [23,27]. Revision surgery for a failed implant due to sensitization is both painful and expensive, and some patients will become sensitized to the implant after the index surgery [10]. Hence, information that improves the ability to predict who will become sensitized is a valuable addition to clinical care.

Currently, information is limited as to the mechanisms leading to a $\mathrm{Ni}^{2+}$-induced immune response in the synovial fluid of a failed implant. Several studies have focused on the MHC-TCR interactions in Ni contact dermatitis, and the type and site of interaction may differ from the interactions leading to $\mathrm{Ni}$ allergy in the joint. The strength of this study lies, in part, in the selection of two patients who had undergone joint replacements with implant failure. Both had CoCrMo implants which also contain nickel. Both patients demonstrated both patch test and LPT reactivity to $\mathrm{Ni}^{2+}$, and we considered them ideal for studying the mechanisms of Ni-induced implant failure.

Our culture conditions also enabled us to select $\mathrm{Ni}^{2+}$-specific $\mathrm{T}$ cell clones in a more efficient way than the typical study, which requires many weeks of $\mathrm{T}$ cell clonal expansion and selection. In our study, we cultured PBMCs with two cycles of Ni stimulation over three weeks, interspersed with one week of hIL-2 culture to maintain cell viability, and then used the CellTrace proliferation dye to select out the proliferating cells specific for $\mathrm{Ni}$. This approach allowed us to more efficiently obtain thousands of paired TCR sequences with much less time and labor. Because antigen presenting cells typically do not survive three weeks of in vitro culture without the addition of growth factors, we added irradiated PBMCs at the beginning of the third week culture to serve as antigen presenting cells without exogenous allergen specific T cell clones. As IFN $\gamma$ is secreted by differentiated Th1 CD4+ and cytotoxic CD8+ cells, it serves as a good marker to detect T cell activation in response to Ni allergen. Notably, the IFN $\gamma$ concentration is much higher by the third week of culture than the first week, indicating enrichment of Ni-specific T cells for both subjects. Under these culture conditions, the percentage of proliferating cells carrying CellTrace fluorescence in the live population is relatively high, $74.1 \%$ for P7 and $53.6 \%$ for P9.

To analyze the TCR repertoire of these Ni-reactive T cells, the cell ranger pipeline from $10 \times$ genomics was used to process Chromium VDJ output, and we analyzed TRAV, TRAJ, TRBV, and TRBJ usage in both CD4+ and CD8+ cells from both subjects. Large-scale paired single cell sequencing of $\mathrm{Ni}^{2+}$ specific $\mathrm{T}$ cells has not been done before. This technique is being increasingly used to identify pathogenic $\mathrm{T}$ cells in many different immune-mediated diseases $[28,29]$. In this study, we showed several highly expanded $\mathrm{T}$ cell clones by sequencing data, and demonstrated the preferential usage of TRBV19, TRBV5-1, and TRBV20-1, consistent with previous studies on $\mathrm{Ni}^{2+}$ contact dermatitis $[11,16,18,20]$. Our study suggests, then, that the certain population of $\mathrm{Ni}^{2+}$-reactive $\mathrm{T}$ cells in joint implant failure may be shared with the $\mathrm{T}$ cells responsible for $\mathrm{Ni}^{2+}$ induced contact dermatitis. TRBV19 usage is the highest in the CD4+ population from P9, but not P7. This suggests the genetic background, e.g., $\mathrm{MHC}$, may have different $\mathrm{Ni}^{2+}$ binding chemistry and select $\mathrm{T}$ cell repertoire. Previous studies have shown the preferential usage of TRBV19 in Ni allergic subjects, and our study confirms its importance. A highly expanded CD4+ clone for P7 is TRAV41-TRBV18, which was detected over 50 . We also noted the high percentage of CD8+ T cells in these proliferative populations, accounting for more than $20 \%$ of the total sequenced cells. Although previous studies have detected $\mathrm{Ni}^{2+}$-reactive CD8+ $\mathrm{T}$ cell clones, they did not elucidate the TCR sequences $[16,30,31]$, and our research thus provides important insights into cytotoxic $\mathrm{T}$ cell function in Ni allergy. The alignment of CDR3 $\beta$ sequences identified the presence of Glu at the last fourth position of CDR3 $\beta$, which could 
be a potential important amino acid in $\mathrm{Ni}^{2+}$ coordination. His and Asp/Glu amino acids are common nickel ligands. In our Ani2.3 TCR/DR52c/mimotope structure, Asp and Gln coordinated $\mathrm{Ni}^{2+}$ [15]. Interestingly, this Glu is conserved in both CD4+ and CD8+ cells sequenced. The overrepresentation of Glu at similar positions may also imply that there may be common $\mathrm{Ni}^{2+}$ presenting self-peptides. In future experiments, we plan to clone these highly expanded $\mathrm{T}$ cell clones and identify $\mathrm{Ni}^{2+}$ presenting MHC and peptides.

\section{Materials and Methods}

\subsection{Subject Selection}

Subjects were enrolled in an IRB approved study (Study number HS-2511, original approval date is 0410 2010, most recent approval date is 2907 2020) at National Jewish Health, and provided informed consent to participate. All samples provided for inclusion in this study were from de-identified subjects and were exempt from IRB review of the University of Colorado. Two patients P7 and P9 were selected for our study on the basis of a positive $\mathrm{Ni}^{2+}$ patch test and positive $\mathrm{Ni}^{2+} \mathrm{LPT}$, as well as a poorly functioning $\mathrm{Ni}^{2+}$ containing implant.

\subsection{PBMC Culture and Stimulation}

PBMC preparation and storage were performed according to previously established methods [18]. In addition, $10^{7}$ PBMC cells were thawed from P7 and P9 patients, cultured in enriched MEM as previously described [32], and supplied with $10 \%$ heat-inactivated premium FBS (VWR, PA, USA, product 97068-085, lot 214B17). Furthermore, $100 \mu \mathrm{M}$ of $\mathrm{NiSO}_{4}$ (Sigma, Germany, product 31483-250G, Lot SZBB0900V) was added to the culture for 7 days. Then, PBMCs were washed twice with BSS, and cultured in media with 20U / mL hIL2 (Sigma, Germany, product 11011456001) for 7 days. Next, PBMCs were washed twice with BSS and returned to media with $100 \mu \mathrm{M} \mathrm{NiSO}_{4}$ and cultured for another 7 days. At this point, PBMCs were stained with CellTrace violet proliferation dye (Invitrogen, City, CA, USA, product C34571) and irradiated PBMCs (40 Gy) from the same patient were added. After 7 days of the second round of $\mathrm{Ni}^{2+}$ stimulation, the proliferative cells were sorted based on CellTrace fluorescence.

\subsection{HLA Genotyping}

$1 \times 10^{6}$ cryopreserved PBMCs were used to isolate the genomic DNA by using the QIAamp DNA Mini-Kit as previously described [33]. The high resolution HLA-DR typing was performed by ClinImmune Labs (Bioscience 2, Aurora, CO, USA).

\subsection{IFN $\gamma$ Measurement}

IFN $\gamma$ was measured using ELISA MAX Standard Set Human IFN- $\gamma$ (Biolegend \#430101).

\subsection{Cell Staining and Single-Cell Sequencing}

The sorted PBMCs were stained based on a Biolegend recommended method for $10 \times$ genomics sequencing. The human TruStain FcXTM Fc blocking reagent (Biolegend Cat\# 422301) was used to block the Fc receptors, and the PBMCs from P7 and P9 patients were stained with TotalSeqTM-C0252 anti-human Hashtag 2 Antibody (Biolegend Cat\# 394663) and TotalSeqTM-C0253 anti-human Hashtag 3 Antibody (Biolegend Cat\# 394665), respectively. TotalSeqTM-C0072 anti-human CD4 Antibody (Biolegend Cat\# 300567) and TotalSeqTM-C0046 anti-human CD8 Antibody (Biolegend Cat\# 344753) were used on both patients to distinguish CD4 and CD8 T cell populations within the sample. After staining, the PBMCs from two patients were merged and 5000 cells were used for single cell V(D)J sequencing by personnel at Genomics and Microarray Core Facility at the University of Colorado Anschutz Medical Campus. There were 40 million reads performed on V(D)J library and 30 million reads on cell hashing/CITE seq library. 


\subsection{Data Processing}

The $10 \times$ genomics "Cell ranger v3.1.0 vdj" function was used to obtain the paired alpha and beta chain VDJ sequences, and "Cell ranger v3.1.0 count" function was used to analyze gene expression and cell hashing. The gene expression was aligned against the human reference (GRCh38) 3.0.0 dataset.

\section{Conclusions}

In summary, this study characterized the $\mathrm{Ni}^{2+}$-reactive $\mathrm{T}$ cell repertoires derived from the peripheral blood of joint failure patients using single-cell sequencing techniques. In addition, 2650 out of 5000 productive TCR sequences were identified, and the preferential usage of TCR alpha and beta chain was analyzed. TRBV19 and TRBV20-1 segments are present in a high percentage of both P7 and P9 sequenced T cells, consistent with previous studies on the $\mathrm{T}$ cell repertoire from contact dermatitis patients. This suggests that skin and joint sensitization processes could share similar pathogenic $\mathrm{T}$ cells. We identified a conserved glutamic acid in the TCR CDR3 $\beta$ sequences in our study, which is a potential nickel binding site. The findings in this study are important for understanding the molecular mechanisms of T-cell mediated nickel hypersensitivity, as well as the development of therapeutics for nickel-induced joint implant failure.

Author Contributions: Conceptualization, S.D., L.C., Y.Z., K.P.; methodology, S.D., L.C., Y.Z.; software, L.C.; writing —original draft preparation, L.C., Y.Z.; writing—review and editing, S.D.; visualization, L.C.; funding acquisition, S.D. All authors have read and agreed to the published version of the manuscript.

Funding: This work was supported by NIH Grants R01ES025797 and R21ES025885.

Institutional Review Board Statement: Subjects were enrolled in an IRB approved study (Study number HS-2511, original approval date is 0410 2010, most recent approval date is 2907 2020) at National Jewish Health.

Informed Consent Statement: All samples provided for inclusion in this study were from deidentified subjects and were exempt from IRB review of University of Colorado.

Data Availability Statement: Data available in a publicly accessible repository.

Acknowledgments: The authors thank the Genomics and Microarray Core at University of Colorado Anschutz Medical Campus. The authors are also grateful to Andrew Fontenot, and Maki Nakayama and Guan Gui for helpful suggestions.

Conflicts of Interest: The authors declare no conflict of interest.

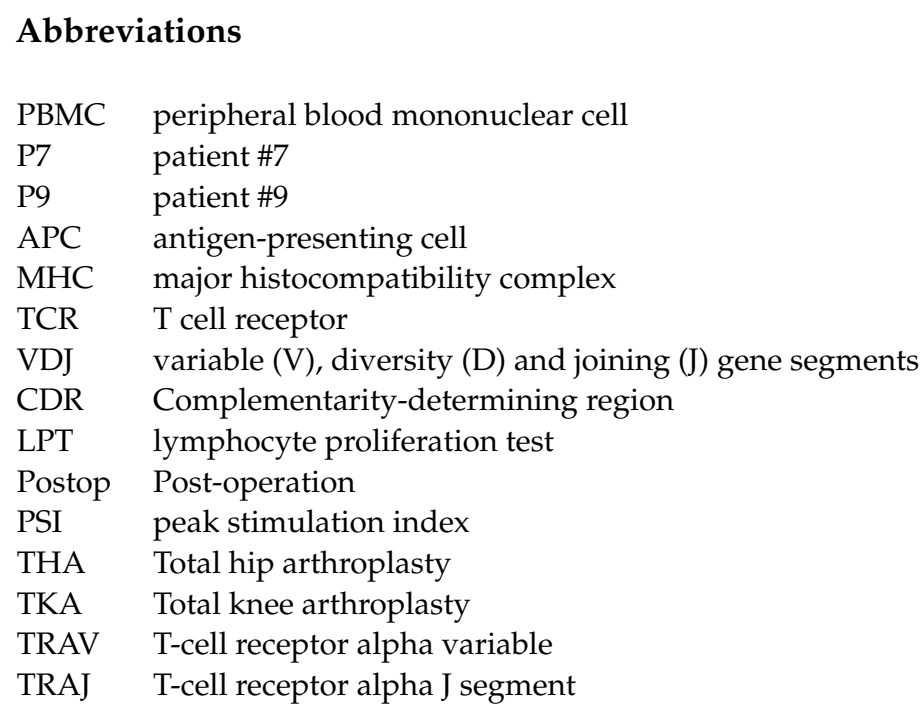


TRBV T-cell receptor beta variable

TRBJ T-cell receptor beta J segment

Th1 Th1 helper cell

Tc1 Type 1 CD8+ T cell

\section{References}

1. Williams, S.N.; Wolford, M.L.; Bercovitz, A. Hospitalization for Total Knee Replacement among Inpatients Aged 45 and Over: United States, 2000-2010. NCHS Data Brief 2015, 186, 1-8.

2. Kurtz, S.; Ong, K.; Lau, E.; Mowat, F.; Halpern, M. Projections of primary and revision hip and knee arthroplasty in the United States from 2005 to 2030. J. Bone Jt. Surg. Am. 2007, 89, 780-785. [CrossRef]

3. Labek, G.; Janda, W.; Agreiter, M.; Schuh, R.; Bohler, N. Organisation, data evaluation, interpretation and effect of arthroplasty register data on the outcome in terms of revision rate in total hip arthroplasty. Int. Orthop. 2011, 35, 157-163. [CrossRef] [PubMed]

4. Ackerman, I.N.; Bohensky, M.A.; Zomer, E.; Tacey, M.; Gorelik, A.; Brand, C.A.; De Steiger, R. The projected burden of primary total knee and hip replacement for osteoarthritis in Australia to the year 2030. BMC Musculoskelet. Disord. 2019, 20, 90. [CrossRef]

5. Pacheco, K.A. Allergy to Surgical Implants. Clin. Rev. Allergy Immunol. 2019, 56, 72-85. [CrossRef] [PubMed]

6. Jakobsen, S.S.; Liden, C.; Soballe, K.; Johansen, J.D.; Menne, T.; Lundgren, L.; Bregnbak, D.; Moller, P.; Jellesen, M.S.; Thyssen, J.P. Failure of total hip implants: Metals and metal release in 52 cases. Contact Dermat. 2014, 71, 319-325. [CrossRef]

7. Schmidt, M.; Raghavan, B.; Muller, V.; Vogl, T.; Fejer, G.; Tchaptchet, S.; Keck, S.; Kalis, C.; Nielsen, P.J.; Galanos, C.; et al. Crucial role for human Toll-like receptor 4 in the development of contact allergy to nickel. Nat. Immunol. 2010, 11, 814-819. [CrossRef]

8. Grabbe, S.; Schwarz, T. Immunoregulatory mechanisms involved in elicitation of allergic contact hypersensitivity. Immunol. Today 1998, 19, 37-44. [CrossRef]

9. Gittler, K.J.; Krueger, J.G.; Guttman-Yassky, E. Atopic dermatitis results in intrinsic barrier and immune abnormalities: Implications for contact dermatitis. J. Allergy Clin. Immunol. 2013, 131, 300-313. [CrossRef]

10. Thomas, P.; Braathen, L.R.; Dorig, M.; Aubock, J.; Nestle, F.; Werfel, T.; Willert, H.G. Increased metal allergy in patients with failed metal-on-metal hip arthroplasty and peri-implant T-lymphocytic inflammation. Allergy 2009, 64, 1157-1165. [CrossRef]

11. Vollmer, J.; Fritz, M.; Dormoy, A.; Weltzien, H.U.; Moulon, C. Dominance of the BV17 element in nickel-specific human T cell receptors relates to severity of contact sensitivity. Eur. J. Immunol. 1997, 27, 1865-1874. [CrossRef]

12. Vollmer, J.; Weltzien, H.U.; Gamerdinger, K.; Lang, S.; Choleva, Y.; Moulon, C. Antigen contacts by Ni-reactive TCR: Typical alphass chain cooperation versus alpha chain-dominated specificity. Int. Immunol. 2000, 12, 1723-1731. [CrossRef]

13. Gamerdinger, K.; Moulon, C.; Karp, D.R.; Van Bergen, J.; Koning, F.; Wild, D.; Pflugfelder, U.; Weltzien, H.U. A new type of metal recognition by human T cells: Contact residues for peptide-independent bridging of $\mathrm{T}$ cell receptor and major histocompatibility complex by nickel. J. Exp. Med. 2003, 197, 1345-1353. [CrossRef] [PubMed]

14. Lu, L.; Vollmer, J.; Moulon, C.; Weltzien, H.U.; Marrack, P.; Kappler, J. Components of the ligand for a Ni++ reactive human T cell clone. J. Exp. Med. 2003, 197, 567-574. [CrossRef] [PubMed]

15. Yin, L.; Crawford, F.; Marrack, P.; Kappler, J.W.; Dai, S. T-cell receptor (TCR) interaction with peptides that mimic nickel offers insight into nickel contact allergy. Proc. Natl. Acad. Sci. USA 2012, 109, 18517-18522. [CrossRef] [PubMed]

16. Bechara, R.; Pollastro, S.; Azoury, M.E.; Szely, N.; Maillere, B.; De Vries, N.; Pallardy, M. Identification and Characterization of Circulating Naive CD4+ and CD8+ T Cells Recognizing Nickel. Front. Immunol. 2019, 10, 1331. [CrossRef]

17. Aparicio-Soto, M.; Riedel, F.; Leddermann, M.; Bacher, P.; Scheffold, A.; Kuhl, H.; Timmermann, B.; Chudakov, D.M.; Molin, S.; Worm, M.; et al. TCRs with segment TRAV9-2 or a CDR3 histidine are overrepresented among nickel-specific CD4+ T cells. Allergy 2020, 75, 2574-2586. [CrossRef]

18. Zhang, Y.; Wang, Y.; Anderson, K.; Novikov, A.; Liu, Z.; Pacheco, K.; Dai, S. Using DR52c/Ni(2+) mimotope tetramers to detect $\mathrm{Ni}(2+)$ reactive CD4(+) T cells in patients with joint replacement failure. Toxicol. Appl. Pharmacol. 2017, 331, 69-75. [CrossRef] [PubMed]

19. Silvennoinen-Kassinen, S.; Karvonen, J.; Ikaheimo, I. Restricted and individual usage of T-cell receptor beta-gene variables in nickel-induced CD4+ and CD8+ cells. Scand. J. Immunol. 1998, 48, 99-102. [CrossRef]

20. Budinger, L.; Neuser, N.; Totzke, U.; Merk, H.F.; Hertl, M. Preferential usage of TCR-Vbeta17 by peripheral and cutaneous T cells in nickel-induced contact dermatitis. J. Immunol. 2001, 167, 6038-6044. [CrossRef]

21. Werfel, T.; Hentschel, M.; Kapp, A.; Renz, H. Dichotomy of blood- and skin-derived IL-4-producing allergen-specific T cells and restricted V beta repertoire in nickel-mediated contact dermatitis. J. Immunol. 1997, 158, 2500-2505.

22. Kou, C.Z.; Puhr, J.S.; Rojas, M.; McCormack, W.T.; Goodenow, M.M.; Sleasman, J.W. T-Cell receptor Vbeta repertoire CDR3 length diversity differs within CD45RA and CD45RO T-cell subsets in healthy and human immunodeficiency virus-infected children. Clin. Diagn. Lab. Immunol. 2000, 7, 953-959. [CrossRef] [PubMed]

23. Atanaskova Mesinkovska, N.; Tellez, A.; Molina, L.; Honari, G.; Sood, A.; Barsoum, W.; Taylor, J.S. The effect of patch testing on surgical practices and outcomes in orthopedic patients with metal implants. Arch. Dermatol. 2012, 148, 687-693. [CrossRef]

24. Foussereau, J.; Laugier, P. Allergic eczemas from metallic foreign bodies. Trans. St. John's Hosp. Dermatol. Soc. 1966, 52, $220-225$.

25. Hallab, N.; Merritt, K.; Jacobs, J.J. Metal sensitivity in patients with orthopaedic implants. J. Bone Jt Surg. Am. 2001, 83, 428-436. [CrossRef] [PubMed] 
26. Symeonides, P.P.; Paschaloglou, C.; Papageorgiou, S. An allergic reaction after internal fixation of a fracture using a vitallium plate. J. Allergy Clin. Immunol. 1973, 51, 251-252. [CrossRef]

27. Adala, R.; Chakravarthy, M.; Srinivas, V.; Pai, S. Orthopaedic surgery in a patient with metal sensitivity. J. Cutan. Aesthet. Surg. 2011, 4, 67-68. [CrossRef]

28. Ostendorf, L.; Burns, M.; Durek, P.; Heinz, G.A.; Heinrich, F.; Garantziotis, P.; Enghard, P.; Richter, U.; Biesen, R.; Schneider, U.; et al. Targeting CD38 with Daratumumab in Refractory Systemic Lupus Erythematosus. N. Engl. J. Med. 2020, 383, $1149-1155$. [CrossRef] [PubMed]

29. Ramesh, A.; Schubert, R.D.; Greenfield, A.L.; Dandekar, R.; Loudermilk, R.; Sabatino, J.J., Jr.; Koelzer, M.T.; Tran, E.B.; Koshal, K.; Kim, K.; et al. A pathogenic and clonally expanded B cell transcriptome in active multiple sclerosis. Proc. Natl. Acad. Sci. USA 2020, 117, 22932-22943. [CrossRef]

30. Traidl, C.; Sebastiani, S.; Albanesi, C.; Merk, H.F.; Puddu, P.; Girolomoni, G.; Cavani, A. Disparate cytotoxic activity of nickelspecific CD8+ and CD4+ T cell subsets against keratinocytes. J. Immunol. 2000, 165, 3058-3064. [CrossRef]

31. Sebastiani, S.; Albanesi, C.; Nasorri, F.; Girolomoni, G.; Cavani, A. Nickel-specific CD4(+) and CD8(+) T cells display distinct migratory responses to chemokines produced during allergic contact dermatitis. J. Invest. Dermatol. 2002, 118, 1052-1058. [CrossRef] [PubMed]

32. White, J.; Kappler, J.; Marrack, P. Production and characterization of T cell hybridomas. Methods Mol. Biol. 2000, 134, 185-193. [PubMed]

33. Moulon, C.; Vollmer, J.; Weltzien, H.U. Characterization of processing requirements and metal cross-reactivities in T cell clones from patients with allergic contact dermatitis to nickel. Eur. J. Immunol. 1995, 25, 3308-3315. [CrossRef] [PubMed] 